\title{
HIDRÓLISIS ENZIMÁTICA DE ALMIDÓN
}

\author{
ENZYMATIC HYDROLYSIS OF STARCH \\ Claudio Raúl Bernal Bustos* \\ Diana Morales** \\ Laura Cuellar**** \\ Sebastián Jaramillo*****
}

\section{Resumen}

Este artículo revisa los avances científicos que se han realizado respecto a la comprensión del fenómeno de hidrolisis parcial del almidón, empleando la enzima glucoamilasa de Aspergillus niger y teniendo en cuenta que esta enzima a nivel estructural tiene un doble dominio de unión (SBD, starch binding domain) que permite, por un lado, su inactivación, y, por el otro, argumentar teóricamente la actividad catalítica en un sustrato amiláceo. El interés de esta revisión, junto a las evidencias de un modelo empírico obtenido a partir de la experimentación y observación y luego del empleo de los datos experimentales en la hidrólisis parcial del almidón de quinua, es establecer los principales avances realizados alrededor del establecimiento de la cinética de la reacción enzimaamilosa en sustratos amiláceos. No obstante, es oportuno aclarar que en este artículo no se presenta lo correspondiente al proceso de inactivación enzimática, así como tampoco lo relacionado a la interacción molecular enzima-amilosa.

Palabras clave: almidón, hidrolisis enzimática, Aspergillus niger, Chenopodium quinoa Willd., sustituto de grasa.

\section{Abstract}

This paper reviews the scientific advances that have been made regarding the understanding of the phenomenon of partial hydrolysis of the starch, using the enzyme Glucoamylase from Aspergillus niger and taking into account that this enzyme; at a structural level has a double (SBD,

\footnotetext{
* Licenciado en Química, magíster en Nutrigenómica y Nutrición Personalizada, magíster en Seguridad y Biotecnología Alimentaria. Estudiante del Doctorado en Avances en Ciencias y Biotecnología Alimentaria (Universidad De Burgos). Director Centro BIQA, docente investigador, Fundación Universidad de América.

Claudio.bernal@investigadores.uamerica.edu.co

** Química, magíster en Ingeniería Química. Docente investigador, Programa de Ingeniería Química, Facultad de Ingeniería, Fundación Universidad de América. Diana.morales@profesores.uamerica.edu.co

*** Estudiante coinvestigador. Centro BIQA, Programa de Ingeniería Química, Facultad de Ingeniería, Fundación Universidad de América. Laura.cuellar@estudiantes.uamerica.edu.co

${ }_{* * * *}$ Estudiante coinvestigador. Centro BIQA, Programa de Ingeniería Química, Facultad de Ingeniería, Fundación Universidad de América. Sebastian.jaramillo@estudiantes.uamerica.edu.co
} 
starch binding domain) binding domain, allowing, on the one hand, its inactivation, and, on the other hand, argues in a starch substrate catalytic activity. The interest of this review, along with the evidence of an empirical model obtained from experimentation and observation after the use of the experimental data in the partial hydrolysis of starch of quinoa, the scope of this review aims to establish major progress made around the establishment of the kinetics of the reaction of enzyme-amylose in starch substrates. However, it is important to clarify that this review does not show what corresponds to the process of enzymatic inactivation and what is related to the enzyme-amylose molecular interaction either.

Keywords: starch, enzymatic hydrolysis, Aspergillus niger, Chenopodium quinoa Willd., fat mimetic.

\section{INTRODUCCIÓN}

El grupo de investigación Centro de Biosprección e Ingeniería Química Aplicada al Estudio de Biomoléculas e Industria (Centro BIQA), de la Fundación Universidad de América, viene realizando aproximaciones experimentales en laboratorio alrededor de la definición de un "patrón de uso" del almidón contenido en los granos-semillas de la especie Chenopodium quinoa Willd., para contribuir a la conformación de la cadena productiva y de valor de la quinua en Colombia (Bernal, Villegas y Sandoval, 2015).

Las evidencias se fundamentan en los estudios de caracterización fisicoquímica del almidón nativo de las variedades colombianas de quinua (Bernal, Raúl, Duarte y Ramírez, 2015), lo que, a su vez, implica la definición de la secuenciación del proceso, que parte de la recolección de las muestras, carácter ex situ, continuando con las etapas de selección, secado, fraccionamiento, caracterización y funcionalización. El proceso de extracción ha involucrado el desarrollo de prototipos mecánicos e industriales (Bejarano, Rodríguez, Guarín y Bernal, 2016), tanto para el acondicionamiento de selección de granos (Jiménéz, Ochoa, Pava y Bernal, 2015), su secado (Martínez, Ochoa, Trujillo y Bernal, 2015) y fraccionamiento, así como para el secado del almidón (Borraez, Mejía Ochoa y Bernal, 2016; González, Morales y Bernal, 2016).

Corresponde informar en este artículo que el Centro BIQA está incursionando particularmente en aplicaciones para la industria alimentaria con el objetivo de reducir las grasas en ciertas matrices estructurales alimentarias. Este objetivo se sustenta en dos premisas: por un lado, la contribución calórica de una grasa es de $9 \mathrm{Kcal} / \mathrm{g}$ respecto a $4 \mathrm{Kcal} / \mathrm{g}$ de un carbohidrato de tipo amiláceo y, por otro, el almidón de quinua presenta un bajo índice glicémico (Abbas, Khalil, Shobirin y Hussin, 2010; Abbas et ál., 2010; Acero y Guzmán, 2015). El almidón de quinua tienen también aplicaciones cosméticas (Chiu y Solarek, 2009; Bernal, Duarte y Ramirez, 2015; Bernal, Cárdenas, Rozo, Daza y Echeverry, 2014; Bernal, Leal y Garzón, 2009; Daza, Bernal y Echeverri, 2012; Pastás, Bernal y Pineda, 2012) y sirve para el desarrollo de nuevas estructuras poliméricas híbridas con potencial biodegradable y biocompatibles (Masina et ál., 2016).

Como objeto final de las investigaciones, el Centro BIQA dedica esfuerzos a la:

[...] ingenierización de los procesos y de productos, promoviendo el uso de los recursos teóricos de los fenómenos cinéticos, sean físicos, químicos o biotecnológicos, para llevar los resultados experimentales a la consolidación de industria, lo que para ello, la ingenierización de procesos y de productos se realizan en cooperación con los programas de Ingeniería Química, Mecánica e Ingeniería Industrial de la Fundación Universidad de América, lo que ha permitido dar inicio al desarrollo de un modelo de enseñanza integrada y bajo una concepción pedagógica fundamentada por (Bernal y Morales, 2016). 
Emplear el almidón de quinua en la definición de un patrón de uso requiere realizar modificaciones en la estructura de este polímero, por lo que este artículo de revisión enfatiza en la definición de las cinéticas, los mecanismos de reacción y los modelos matemáticos propuestos para llevar a cabo procesos de ingenierización futuros.

\section{Metodología}

La revisión bibliográfica en las bases de datos de Scopus, Ebsco Host, Knovel, Virtual Pro, entre otras, ${ }^{1}$ estableció las siguientes palabras clave como principales descriptores de búsqueda del periodo 2011-2016: Quinoa Starch Hydrolysis, Enzymatic Hydrolysis Starch, Enzymatic Kinetic Starch Hydrolysis, starch and amylose and hydrolysis. La revisión dio como resultado la definición de modelos de la hidrólisis enzimática total de almidón y determinó que existe un vacío temático alrededor de la hidrólisis parcial de almidón y la definición de cinéticas de esta situación.

\section{Resultados}

\section{Almidón}

El almidón es el principal polisacárido de reserva de la mayoría de los vegetales; se encuentra principalmente en cereales, legumbres, pseudocereales y algunas frutas tropicales. Un almidón está constituido por dos macromoléculas de estructura diferente: la amilosa y la amilopectina; estas moléculas tienen un alto peso molecular, están organizadas en gránulos semicristalinos (entre $1 \mathrm{a}$ $100 \mu \mathrm{m})$ y son un factor influyente en las propiedades sensoriales y reológicas, principalmente en su capacidad de hidratación y gelatinización.

La amilosa es un polímero de cadena lineal, tiene una estructura amorfa, está presente como un complejo helicoidal con una estructura lineal de D-glupiranosa unido por enlaces $\alpha-(1 \rightarrow 4)$ y la maltosa es la unidad de disacárido que se repite. El peso molecular de la amilosa varía según la fuente botánica y el método de aislamiento. La amilopectina presenta un mayor peso molecular, tiene parte de su cadena externa como dobles hélices con una estructura principal de $\alpha$-D-glucosa unidas por enlaces $\alpha-(1 \rightarrow 4)$ y ramificaciones de cadenas laterales cortas unidas con enlaces $\alpha-(1 \rightarrow 6)$ cada 24 o 30 residuos de glucosa.

Teniendo en cuenta las características y organización de los gránulos de almidón, el $70 \%$ aproximadamente de la masa de un grano de almidón se considera amorfo y el $30 \%$ es aproximadamente cristalino. En las zonas amorfas se localiza la mayor parte de amilosa, aunque también coexiste una fracción considerable de amilopectina; no obstante, las zonas cristalinas están formadas predominantemente por amilopectina

En cuanto a estas diferencias estructurales, la amilopectina tiene una estructura más estable y es más difícil realizar la ruptura de sus enlaces, hecho por el que se degrada de una manera más lenta que la amilosa (Chiu y Solarek, 2009), razón por lo que la hipótesis enzima-amilosa (especificidad de la enzima) fundamenta la investigación del CBIQA.

\section{Hidrólisis enzimática}

La estructura del almidón se puede degradar con el empleo de enzimas del tipo endoamilasas, exoamilasas, enzimas desramificadoras y transferasas. Los productos finales encontrados por tipo de enzima han sido diversos. Por ejemplo, para el caso de las endoamilasas, enzima $\alpha$-amilasa, los productos finales son oligosacáridos y dextrinas. Para enzimas exoamilasas (glucoamilasas y

${ }^{1}$ Sistema de biblioteca Baldomero Sanín Cano, Fundación Universidad de América, 2017. 
$\alpha$-glucosidasa), el producto es glucosa o maltosa, etc. No obstante, la hidrólisis enzimática del almidón depende de la estructura granular del almidón, el tamaño y tipo de cristal, la relación amilosa/amilopectina y el peso molecular promedio de dicha relación, además de los cuerpos proteicos y lipídicos y las condiciones de especificidad de la enzima. En la tabla 1 se presentan las principales diferencias estructurales del almidón de diferentes fuentes, utilizados principalmente en el estudio de hidrólisis enzimática.

Tabla 1. Diferencias estructurales de tipo de almidón

\begin{tabular}{|c|c|c|c|c|}
\hline Almidón & $\frac{\text { Diámetro }}{\text { Partícula }(\mu \mathrm{m})}$ & $\begin{array}{c}\text { Amilosa/ } \\
\text { amilopectina (\%) }\end{array}$ & $\begin{array}{l}\text { Patrón de } \\
\text { cristalinidad }\end{array}$ & $\begin{array}{c}\text { Tamaño de cristal } \\
\text { Forma }\end{array}$ \\
\hline Papa normal a & $20-110$ & $20.1-31 / 80$ & B & \\
\hline Maíz normal b & $\begin{array}{l}\text { 1-7 pequeños, } 15-20 \\
\text { largos. }\end{array}$ & $22,4-32,5 / 75$ & A & $\begin{array}{l}\text { Esférica, oval y con } \\
\text { predominio de poligonal }\end{array}$ \\
\hline Arroz normal ${ }^{b}$ & $3-5$ & $5-28.4 / 81$ & A & Angular \\
\hline Trigo normal ${ }^{\mathrm{b}}$ & $2-38$ & $18-30 / 75$ & A & Lenticular esférico \\
\hline Banana (Gros Michel) ${ }^{b}$ & $10-20$ longitud $35-70$ & 22.76 & $\mathrm{C}$ & ----- \\
\hline Yuca (MBra 383) $^{\mathrm{b}}$ & $10-100$ & 14.23 & B & ---- \\
\hline Quinua $^{\mathrm{c}}$ & $1-3$ & $10-21.2 / 85$ & A & Poligonal \\
\hline
\end{tabular}

Nota. a) Raghunathan, Hoover, Waduge, Liu, y Warkentin (2017); b) Shevkani, Singh, Bajaj y Kaur (2017); c) Li, Wang, y Zhu (2016a).

Las enzimas que le interesan a esta investigación son las que degradan el almidón-granular a una temperatura moderada y por debajo de la temperatura de gelatinización (Uthumporn, Shariffa y Karim, 2012). Estas enzimas (EC 3.2.1.1) son las glucoamilasas o 1,4- $\alpha$-D-glucan-glucohidrolasas, cuya exoactividad fractura los enlaces 1.6- y 1.4-, y algunos 1.3-glocosídicos de $\alpha$-glucanos, que generan glucosa a partir de enlaces terminales no reductores de la cadena de almidón y que, por lo tanto, son enzimas sacarificantes. Por otra parte, se debe tener en cuenta que coexiste una relación estructura-función en los componentes del almidón, amilosa-amilopectina; componentes que entran en interacción con los sitios activos que dispone la enzima (glucoamilasa). El estudio de Morris, Gunning, Faulds, Williamson y Svensson (2005) sobre interacción enzima-almidón logró generar un modelo complejo amilosa (SBD, sigla en inglés). Este modelo tiene fundamento en la geometría de los "sitios" de unión; estos sitios pueden ser detectados a través de técnicas que ofrece la microscopía de fuerza atómica (MFA). Como lo manifiesta el trabajo de Morris et ál. (2005), la interpretación preliminar de las estructuras fueron presentadas por Benavent-Gil y Rosell (2017) (Gunning et ál., 2003) Las imágenes de la técnica MFA (100 nm) durante la amilosis detectan varios situaciones en la interacción proteína-amilos que no serán expuestas en este artículo; sin embargo, son consideraciones a explicar en la definición de las velocidades de reacción, los mecanismos de reacción y la cinética enzimática.

La investigación de Li, Wang y Zhu (2016) y un análisis de correlación de Pearson revelan que las propiedades relacionadas a la gelatinización, como el poder de hinchamiento, el índice de solubilidad en agua, la critalinidad, la claridad de la pasta y las propiedades térmicas, son muy afectadas por el perfil de la cadena de amilopectina y el contenido de amilosa. Li, Wang y Zhu (2016) establecen otras propiedades, como la susceptibilidad enzimática y distribución de tamaño de partícula, que también están fuertemente correlacionadas con la composición del almidón y estructura interna de la amilopectina. Jaiswal y Chibbar (2017) resaltan la importancia del tamaño del gránulo de almidón y de la forma y estructura de la amilopectina, y manifiestan que estas son las principales 
características que influencian las propiedades y uso del almidón; igualmente, los autores señalan que la estructura de la cadena de amilopectina es usada para predecir las fuentes botánicas.

Actualmente, se tiene conocimiento sobre la hidrólisis enzimática del almidón para diferentes almidones de fuentes distintas; alguno de estos ya cuenta con una cinética enzimática definida que se realiza de manera industrial. Se ha experimentado con diferentes métodos para la cuantificación de azúcares hidrolizados, ya sea por cromatografía liquida, espectrofotometría, DNS o método Fehling, Tollens y Benedict.

\section{Cinética enzimática}

En los siete artículos seleccionados en la revisión bibliográfica se identificó nueve almidones: papa (solución Panreac 1 \%), maíz, banano, yuca, sorgo, arroz, trigo, quinua y amaranto.

\section{Almidón de papa (solución 1 \% Panreac Química S.L.U)}

El almidón fue sometido a una hidrólisis total con glucoamilasa comercial a un pH de 5.0 y una temperatura de $50{ }^{\circ} \mathrm{C}$, que son las condiciones óptimas de operación de esta enzima; también se realizó el procedimiento a $40{ }^{\circ} \mathrm{C}$ para ver su comportamiento. No se menciona el uso de un diseño experimental ni los criterios para elegir las variables. La experimentación se llevó a cabo en un reactor discontinuo, camisado, agitado y termostatizado de $250 \mathrm{ml}$ y se tomaron 4 concentraciones diferentes de sustrato $(10,15,20$ y $25 \mathrm{~g} / 1)$ y 5 relaciones de enzima/sustrato $(0.6,1.2,2.4,3.6$ y $6.0 \mathrm{~g}$ de enzima/100 g de sustrato) como variables de estudio. Se determinó la variación de la concentración de la enzima con respecto al tiempo; se obtuvo que a $50{ }^{\circ} \mathrm{C}$ el comportamiento se pudo ajustar a Michaelis-Menten, pero a $40^{\circ} \mathrm{C}$ el comportamiento varía. Los datos obtenidos se ajustaron a una ecuación de velocidad de reacción para conversiones menores al $40 \%$; a una mayor conversión se evidencia un comportamiento diferente (Gonzalez, Camacho, Jurado y Sánchez, 1990).

\section{Almidón de maíz}

En otro estudio se empleó como sustrato el almidón de maíz (Zea mayz) aislado de la semilla madura para realizar la hidrólisis total con $\alpha$-amilasa (PPA) y amiloglucosidasa (AAG). Se realizó a un $\mathrm{pH}$ de 6.9 y a $37^{\circ} \mathrm{C}$ para PPA y $55^{\circ} \mathrm{C}$ para $\mathrm{AAG}$, con $10 \mathrm{mg}$ de sustrato (no se especifica el volumen). El diseño experimental utilizó Anova mediante la prueba de Tukey y el análisis de correlación de Pearson con SPSS16.0 Statistical Software Program. Se determinó la cantidad de azúcares liberados durante 72 horas y se compararon los resultados obtenidos para cada enzima. La cinética de la hidrólisis del almidón se expresó como el porcentaje de almidón degradado a partir del almidón añadido durante el período tiempo y en forma no lineal con un modelo de dos parámetros. Para las dos enzimas, la hidrólisis mostró dos fases: una tasa rápida al inicio seguida de una fase de disminución progresiva. Por su parte, Ma, Cai, Wang y Sun (2006) indican que los granos muy finos pueden actuar como grasas "miméticas" y generan una sensación de suavidad como un fenómeno reologico. Lin et ál. (2016) ensayaron con cuatro enzimas hidrolíticas, alfa-amilasa, beta amilasa, glucoamilsa y una dextrosima, para la hidrólisis del almidón de maíz, y encontraron que el tamaño de partícula está entre 2-4 $\mu \mathrm{m}$.

\section{Almidón de banano}

Almidones gelatinizados del banano (Musa paradisiaca) fueron sometidos a un proceso de hidrólisis total por medio de pululanasa OPTIMAX ${ }^{\circledR}$ L-1000 y L-400. El proceso fue llevado a cabo a $4.35 \mathrm{de} \mathrm{pH}$ y varió la temperatura de las experimentaciones a 58 y $62{ }^{\circ} \mathrm{C}$ en un baño de María 
con agitación constante. Se tomaron como variables de estudio la temperatura, la dosis de enzima y el pH. La concentración de sustrato fue de $50 \mathrm{~g} / \mathrm{L}$ y la concentración de la enzima empleada fue de 0.2 y 0.6 g de enzima por gramos de sustrato. El diseño experimental empleado fue un factorial exploratorio de dos niveles, que usó Minitab $15^{\mathrm{TM}}$ versión estudiantil, ANOVA $2 \mathrm{k}$, en base a los resultados óptimos del estudio cinético. El modelado de la hidrólisis enzimática se realiza por Michaelis-Menten, después de hacer una purificación previa de las enzimas comerciales mediante la técnica de FPLC. La prueba estadística de ajuste del modelo Chi cuadrado reveló que el modelo no ajusta adecuadamente los datos experimentales, debido a una subestimación de las incertidumbres experimentales, aunque el modelo constituye, en sí, una buena representación fenomenológica del esquema de reacción planteado.

\section{Almidón de yuca}

El almidón de yuca (Manihot esculenta) también fue usado como materia prima en la hidrólisis total del mismo almidón para la obtención de glucosa. El tipo de enzima utilizada para el procedimiento fue la alfa-amilasa BAN 480 de Bacillus amyloliquefaciens y la amiloglucosidasa obtenida de Aspergillus niger (AMG 300 L de Novo Nordisk). Para la experimentación se adecuó el pH de las muestras a 6 y 4.8 de $\mathrm{pH}$, ajustándolo con una solución buffer de fosfato $0.1 \mathrm{M}$ o con cloruro de calcio y a una temperatura de $70{ }^{\circ} \mathrm{C}$. Se tomaron en cuenta como variables la enzima $(0.1 \mathrm{~g}$ de enzima/100 g de sustrato para cada una), la temperatura, el $\mathrm{pH}$ y las concentraciones de sustrato. La concentración del sustrato se mantuvo constante a $30 \% \mathrm{p} / \mathrm{p}$. Los datos cinéticos fueron ajustados al modelo enzimático de Michaelis-Menten, que evidenció que todos eran procesos de primer orden y que la velocidad de reacción era proporcional a la concentración del reaccionante.

\section{Almidón de sorgo}

El almidón de sorgo (Sorghum) fue estudiado a nivel cinético. Se tomó dos tipos de sorgo procedentes de cultivos de Argelia, con un contenido aproximado de $55.5 \%-75.2 \%$, y se usó tres métodos diferentes de extracción. Los almidones fueron hidrolizados totalmente por medio de glucoamilasa (Sigma Aldrich) en un medio de $4.5 \mathrm{de} \mathrm{pH}$, con una temperatura de $\operatorname{los} 8^{\circ} \mathrm{C}$ y $58^{\circ} \mathrm{C}$ y un volumen de $50 \mathrm{~mL}$. Se contemplaron como variables experimentales el contenido de amilosa, la solubilidad del agua, el poder de hinchamiento y el grado de hidrólisis. Se utilizaron siete concentraciones de sustrato diferentes $(0.65,1.25,2.5,5,7.5,10$ y $20 \mathrm{~g} / \mathrm{l})$ y una para la enzima. El proceso fue bosquejado por medio del método de Michaelis-Menten, con el que se obtuvieron mejores resultados en cuanto a la cantidad de almidón extraído mediante el método del ácido láctico, comparado con los dos métodos restantes (ácido sulfúrico e hidróxido de sodio); además, se demostró que hay mejores rendimientos a mayor concentración. Se concluyó que el método de extracción escogido para el almidón afecta su cinética. Los resultados de las constantes de Michaelis-Menten fueron contrarias para cada tipo de sorgo (Souilah, Boudries, Djabali, Belhadi y Nadjemi, 2015).

\section{Almidón de arroz}

En el estudio del almidón de arroz (Oryza sativa) de Calbiochem Company se realizó un análisis cinético a partir de una ecuación empírica de carácter exponencial. El análisis se hizo a partir de glucoamilasa de Bacillus Licheniformis a condiciones de 6.5 de $\mathrm{pH}$, a $60^{\circ} \mathrm{C}$, en un reactor tipo Batch y con un volumen de $500 \mathrm{~mL}$. Las concentraciones a las cuales fueron estudiadas las muestras de almidón fueron de 1, 2,4 y $8 \%(\mathrm{w} / \mathrm{v})$ y la de enzima de $130500 \mathrm{U} / \mathrm{L}$ o también $5 \mathrm{~mL} / \mathrm{L}$. Durante el proceso experimental se realizaron gráficas donde se evaluó la concentración de almidón en el tiempo (10 min). La glucosa se usó como químico de inhibición. Se observó que a medida que 
aumentaba la concentración de almidón disminuyó el grado de azúcares reducidos. Se usó a una ecuación empírica para la construcción de las curvas de calibración. Se calcularon las constantes de Michaelis-Menten y se analizaron los tres químicos de inhibición (glucosa, maltosa y glicerol). El estudio concluyó que los tres químicos actuaron de manera efectiva e igual como inhibidores de la glucoamilasa. Finalmente, se demostró que una ecuación simple de carácter exponencial representa adecuadamente la información recolectada (Apar y Özbek, 2007).

\section{Almidón de trigo}

El almidón de trigo (Triticum) extraído por licuefacción fue estudiado para la sacarificación enzimática; el análisis utilizó glucoamilasa y varió el pH entre 4 y 5 a $30{ }^{\circ} \mathrm{C}$ (también se realizó un experimento a $55^{\circ} \mathrm{C}$ ). Este se llevó a cabo en $100 \mathrm{ml}$ con una concentración de sustrato entre 12 a $18 \mathrm{~g} / \mathrm{dm}^{3}$ y una relación enzima-sustrato de 1 a $1.5 \mathrm{~mm} 3 / \mathrm{g}$ de sustrato. Se analizó el efecto de la temperatura y el $\mathrm{pH}$ sobre la sacarificación; no se menciona el uso de un diseño experimental. Los parámetros cinéticos a $30^{\circ} \mathrm{C}$ se determinaron por medio de un método no lineal y ajustado a la modificación hecha por los autores del modelo presentado por Akerberg, Zacchi, Torto y Gorton (2000).

\section{Almidón de quinua}

La especie Chenopodium quinoa Willd. se cultiva en la cordillera de los Andes. Esta especie tiene una gran importancia nutricional y funcional. La literatura la ha referenciado como un pseudocereal por el tipo de cristalinidad que tiene su almidón, tipo A, que es el mismo tipo de los cereales (Geera, Nelson, Souza y Huber, 2006). El principal uso de los granos de quinua es la elaboración de productos que equivalen a los desarrollados a partir de trigo, cebada, avena, maíz, etc., como harinas, panes, galletas, fideos, hojuelas, granolas, etc. (Nowak, Du y Charrondière, 2016).

El grano-semilla de quinua madura (de aquí en adelante se denominará con el término "grano") presenta una estructura y morfología particular: tiene un tipo campilótropo o encorvado y es un óvulo curvado en forma tal que la calaza se halla próxima al funículo.

Prego, Maldonado y Otegui (1998) identificaron, en un corte longitudinal medio de la semilla madura, el endospermo, el perisperma y el embrión (Varriano y Defrancisco, 1984). El endospermo está presente únicamente en la región microcapilar y está formado por una o dos capas de células que envuelven el eje radicular del embrión. Por su parte, el embrión está constituido por el eje radicular y dos cotiledones. El perisperma está formado de células de paredes delgadas y uniformes, las cuales son depositarias de granos de almidón de forma angular (Leach y Schoch 1960; Varrianoy Defrancisco, 1984); su tamaño aproximado está entre 1.0 a $2.0 \mu \mathrm{m}$ (Atwell, Patrick y Johnson, 1982), 0.5 a $1.0 \mu \mathrm{m}$ (Prego et ál., 1998) y $1.2 \mu \mathrm{m}$ a $1.8 \mu \mathrm{m}$ para variedades colombianas (Bernal, Duarte y Ramírez, 2015). Los gránulos pueden estar agrupados en agregados amiláceos que tienen un tamaño aproximado de 20 a $25 \mu \mathrm{m}$ (Prego et ál., 1998).

Visto en microscopia electrónica (SEM, scanning electron microscopy), el grano de almidón de quinua empaqueta dos polisacáridos básicos: amilopectina y amilosa. El almidón nativo es caracterizado por el ratio del contenido de amilopectina (de orden ramificada) a amilosa (de orden lineal). La ramificación de amilopectina permite el crecimiento de altos pesos moleculares dentro del gránulo, usualmente es mayor que $500000000 \mathrm{~g} / \mathrm{mole}$; la amilosa es de menor peso molecular que la amilopectina, y por lo general está en el orden de $1000000 \mathrm{~g} / \mathrm{mole}$ (Desmond y Gruber, 1999).

El tamaño pequeño y la uniformidad del gránulo del almidón de quinua dan una textura lisa y una sensación de suavidad. Este atributo le ha interesado particularmente a las industrias de alimentos, de papel y cosmética. Atwell, Patrick y Johnson (1982) realizaron un análisis profundo del 
almidón de quinua que indicó que el contenido de amilosa es del $11 \%$ (valor que es considerado bajo respecto a otros almidones).

Li et ál. (2016) estudiaron las propiedades fisicoquímicas del almidón de quinua en 26 muestras comerciales y analizaron particularmente el poder de hinchamiento (SP, swelling power). Ahamed, Singhal, Kulkami y Palb (1996) encontraron dos estadios en el hinchamiento en el rango de temperatura de $65-95^{\circ} \mathrm{C}$. Otras de las propiedades estudiadas fueron: índice de solubilidad (WSI, water solubility index), amilosa (AML, amylosa leaching), la susceptibilidad enzimática, la claridad de la pasta, las propiedades térmicas, texturales y el contenido aparente de amilosa (AAM, apparent añilese contents). El contenido de amilosa se encuentran en un rango de 7.7 a $25.7 \%$. La variable AAM, en un análisis de correlación, indica que es el factor mayormente relacionado a AML, WSI y los parámetros de la pasta.

El tamaño de gránulo y el contenido de amilosa son factores importantes para la determinación de las propiedades funcionales de cualquier sistema, sea alimenticio, farmacéutico, cosmético, etc. Los estudios sobre las propiedades fisicoquímicas del almidón de quinua se han investigado de manera suficiente por Atwell y Johnson (1982), Varriano y Defrancisco (1984), Lorenz (1990) y Ando et ál. (2002). La viscosidad es relacionada con el ratio de amilosa a amilopectina: a mayor ratio, mayor efecto de espesamiento del polisacárido.

Las propiedades fisicoquímicas del almidón cambian según la variedad de quinua en un rango amplio (Li, Wang y Zhu, 2016; Li y Zhu, 2017; Steffolani, Leon y Perez, 2013). Otra de las propiedades relevantes es la tasa de amilosa/amilopectina y la temperatura de gelatinización, que según la literatura reportan valores de $12.35 \%$ y $55-70{ }^{\circ} \mathrm{C}$, respectivamente. El almidón extraído de las variedades de quinua colombianas reporta un tamaño por gránulo (analizado por MEB) entre $1.12 \mu \mathrm{m}$ a $1.8 \mu \mathrm{m}$ y un promedio de $1.2 \mu \mathrm{m}$. Adicionalmente, se reporta un valor de $50 \%$ de almidón en base seca, $12.8 \%$ de amilosa y un porcentaje de cristalinidad de $16.176 \%$ (Bernal et ál., 2015). Esto comprueba que esta propiedad del almidón se ve afectada por la fuente botánica o método de extracción; pese a esto, los gránulos de almidón de quinua muestran una forma poligonal irregular para todos los datos reportados en la literatura (Bernal et ál, 2015)

El almidón de quinua ha sido sometido a una hidrólisis parcial con glucoamilasa de $A s$ pergillus niger, teniendo en cuenta que, a nivel estructural, esta enzima tiene SBD. También se realizaron ensayos con otras enzimas hidrolíticas para la depolimerización del almidón de quinua, con el objeto de desdoblar las conformaciones anomericas (Acero y Guzmán, 2015). Se fijó la temperatura y el $\mathrm{pH}$ de $30^{\circ} \mathrm{C}$ a $37^{\circ} \mathrm{C}$ y de 4.0 a 4.7 , respectivamente. Para la hidrólisis, se siguió un diseño experimental $3 \mathrm{k}$ y se tomó como variables la concentración de almidón, la concentración de encima y el tiempo del procedimiento. Las concentraciones de enzima fueron $7.59 \mathrm{E}-4,1.57 \mathrm{E}-3$ y $2.47 \mathrm{E}-3$, y las de almidón fueron 10,15 y $20 \%$ p/p. E1 análisis de ANOVA realizado sobre los resultados obtenidos muestra las diferentes interacciones entre las variables respecto al menor tamaño de gránulo por medio del uso del programa MiniTab 17. Se logró una disminución del $60 \%$ del tamaño de gránulo respecto al almidón sin modificar; con el almidón hidrolizado se realizó una sustitución de hasta el $60 \%$ en una matriz de mayonesa que conservó las propiedades reológicas de la misma.

\section{Conclusiones}

Como resultado de la revisión se encontró, para la fecha de los análisis, que en siete estudios se ha determinado la cinética de hidrólisis enzimática para diferentes almidones. En todas las investigaciones se estudió la degradación completa del almidón, con lo que se obtuvo los parámetros cinéticos; para el caso del almidón de quinua, se obtuvo un modelo empírico de degradación de la 
fracción amilosa. No se encontraron referencias acerca de cinéticas y los mecanismos de reacción de hidrolisis parciales de almidones, menos aún que se haya realizado en el almidón de quinua, que es el objeto de investigación del Centro BIQA.

\section{Agradecimientos}

E1 Centro BIQA agradece al Sistema de Bibliotecas Baldomero Sanín Cano, por las contribuciones respecto a la información suministrada. A la Fundación Universidad de América, en cabeza de sus directivas, al igual que al Departamento de Investigación de la Universidad, por todas las contribuciones otorgadas.

\section{Referencias}

Abbas, K., Khalil, , Shobirin, A., y Hussin, M. (2010). Modified Starches and Their Usages in Selected Food Products: A Review Study. Journal of Agricultural Science, 2(2), 90-100.

Acero, J., y Guzmán, A. (2015). Hidrolisis enzimatica de almidón nativo de quinua, para obtener un producto con propiedades reologicas de una grasa alimentaria (tesis de pregrado). Facultad de Ingeniería Química, Fundación Universidad de América, Bogotá, Colombia.

Ahamed, N., Singhal, R., Kulkami, P., y Palb, M. (1996). Physicochemical and functional properties of Chenopodium qrinoa starch. Carbohydrate Polymers, 31(96), 99-103.

Akerberg, C., Zacchi, G., Torto, N., y Gorton, L. (2000). A kinetic model for enzymatic wheat starch saccharification. Journal of Chemical Technology and Biotechnology, 75(4), 306-314. https://doi. org/10.1002/(SICI)1097-4660(200004)75:4<306::AID-JCTB218>3.0.CO;2-U

Ando, H., Chen, Y.-C., Tang, H., Shimizu, M., Watanabe, K., y Mitsunaga, T. (2002). Food Components in Fractions of Quinoa Seed. Food Science and Technology Research, 8(1), 80-84. https://doi.org/10.3136/fstr.8.80

Apar, D., y Özbek, B. (2007). Estimation of Kinetic Parameters for Rice Starch Hydrolysis Inhibited By Added Materials. Chemical Engineering Communications, 194(3), 334-344. https:// doi.org/10.1080/15397730600830039

Atwell, W., Patrick, B., Johnson, L., y Glass, R. (1982). Characterization of Quinoa Starch. Cereal Chemistry, 60(1), 9-11.

Bejarano, J., Rodríguez, L., Guarín, A., y Bernal, C. (2016). Diseño y simulación a escala de banco, de un modelo matemático del proceso de extracción de almidón nativo de la especie Chenopodium quinoa (WILLD) variedades colombianas (tesis de pregrado). Facultad de Ingeniería Industrial, Fundación Universidad de América, Bogotá D. C., Colombia.

Benavent-Gil, Y., y Rosell, C. (2017). Comparison of porous starches obtained from different enzyme types and levels. Carbohydrate Polymers, 157, 533-540. https://doi.org/10.1016/j. carbpol.2016.10.047

Bernal, C., Leal, A., y Garzón, J. (2009). Obtención, a escala de laboratorio, de octenilsuccinato alumínico de almidón de quinua, con miras a su utilización en un producto cosmético. $R e-$ vista Virtual Pro, 95, 15-34.

Bernal, C., y Morales, G. (2016). Modelo pedagógico para la interactividad de las ciencias técnicas. Aproximación conceptual. Revista de Investigación, 9(2), 106-113. 
Bernal, C., Duarte, P., Ramírez, L., y Acero, J. (2015). Quinua, Chenopodium quinua (Willd.), en Colombia. Caracterizacion de gránulos de almidón nativo de quinua por IR-ATR, MEB, DRX. Revista de Investigación, 8(2), 122-131

Bernal, C., Cárdenas, O., Rozo, W., Daza, P., y Echeverry, A. (2014). Octenilsuccinato alumínico de almidón de quinua -grado cosmético- $\mathrm{y}$ su certificación bajo la denominación de ingrediente natural. Revista de Investigación, 7(2), 38-49.

Bernal, C., Villegas E., Sandoval, G., Lache, A., y Correa, C. (2015). Quinua (Chenopodium quinoa Willd.) en Colombia. Primera Entrega: un nuevo enfoque de negocio. Revista Virtual Pro, 164, 1-31.

Borraez, C., Mejía, N., Ochoa, J., y Bernal, C. (2016). Desarrollo de una tecnologia escala de banco para el secado de crudo de almidón nativo de quinua (tesis de pregrado). Facultad de Ingeniero Mecánica, Fundación Universidad de América, Bogotá D. C., Colombia.

Chiu, C., y Solarek, D. (2009). Modification of Starches. En J. BeMiller y R. Whistler (Eds.), Starch : Chemistry and Technology (3a ed., pp. 629-655). EE. UU.: Elsevier.

Chiu, C., y Solarek, D. (2009). Modification of Starches. En J. BeMiller y R. Whistler (Eds.), Starch: Chemistry and Technology ( $3^{\mathrm{a}}$ ed., pp. 629-655). EE. UU.: Elsevier.

Daza, P., Bernal, C., y Echeverri, A. (2012). Evaluación del proceso de obtención de octenilsuccinato aluminico de almidón de Quinua-Osa Quinua, grado cosmético, para la obtención del producto apto para su certificación (tesis de pregrado). Facultad de Ingeniería Química, Fundación Universidad de América, Bogotá, D. C., Colombia.

Desmond, E., y Gruber, J. (Eds.). (1999). Principles of Polymer Science and Technology in Cosmetics and Personal Care. Nueva York: Marcel Dekker.

Duarte, P., Bernal, C., y Ramirez, L. (2015). Obtención de carboximetil almidón de quinua, a escala laboratotio, a partir de almidón nativo de Chenopodium quinoa (Willd.) para su uso en la industria de bidrocarburos comoa ditivo en fluidos de perforación (tesis de pregrado). Facultad de Ingeniería Química, Fundación Universidad de América. Bogotá D. C., Colombia.

Geera, B., Nelson, J., Souza, E., y Huber, K. (2006). Composition and properties of A- and B-type starch granules of wild-type, partial waxy, and waxy soft wheat. Cereal Chemistry, 83(5), 551571. https://doi.org/10.1094/CC-83-0551

Gunning, A., Giardina, T., Faulds, C.,Juge, N., Ring, S., Williamson, G., y Morris, V. (2003). Surfactant-mediated solubilisation of amylose and visualisation by atomic force microscopy. Carbohydrate Polymers, 51(2), 177-182.

González, P., Camacho, F., Jurado, E. y Sanchez, R. (1990). Cinética de la hidrolisis de almidon soluble con glucoamilasa. Anales de Quimica, 86(7), 728-732.

González, A., Juan, P., Morales, M., y Bernal, C. (2016). Desarrollo tecnológico a escala laboratorio de un sistema de mezclado y filtrado de crudo de almidón proveniente de granos semilla de la especie Chenopadium Quinua (Willd.) (tesis de pregrado). Facultad de Ingeniería Meánica, Fundación Universidad de América, Bogotá D. C., Colombia.

Jaiswal, S., y Chibbar, R. (2017). Amylopectin small chain glucans form structure fingerprint that determines botanical origin of starch. Carbohydrate Polymers, 158, 112-123. https://doi.org/ http://dx.doi.org/10.1016/j.carbpol.2016.11.059 
Jiménéz, J., Ochoa, J., Pava, S., y Bernal, C. (2015). Diseño de una máquina clasificadora, por dimensiones, de la semilla de quinua usando una energía alternativa (tesis de pregrado). Facultad de Ingeniería Mecánica, Fundación Universidad de América, Bogotá D. C., Colombia.

Leach, H, y Schoch, T. (1960). Structure of the starch granule II: action of various amylases on granular starches. Cereal Chemistry, 38(1), 34-46.

Li, G., Wang, S., y Zhu, F. (2016). Physicochemical properties of quinoa starch. Carbohydrate Polymers, 137, 328-338. https://doi.org/10.1016/j.carbpol.2015.10.064

Li, G., y Zhu, F. (2017). Amylopectin molecular structure in relation to physicochemical properties of quinoa starch. Carbohydrate Polymers, 164, 396-402. https://doi.org/http://dx.doi. org/10.1016/j.carbpol.2017.02.014

Lorenz, K. (1990). Quinoa (Chenopodium quinoa) Starch-Physico-chemical Properties and Functional Characteristics. Starch-Stärke, 42(3),81-86. https://doi.org/10.1002/star.19900420302

Ma, Y., Cai, C., Wang, J., y Sun, D. (2006). Enzymatic hydrolysis of corn starch for producing fat mimetics. Journal of Food Engineering, 73, 297-303. https://doi.org/10.1016/j. jfoodeng.2005.01.023

Martínez, J., Ochoa, J., Trujillo, A., y Bernal, C. (2015). Diseño de una máquina para el secado de la semilla de quinua usando una energía alternativa (tesis de pregrado). Facultad de Ingeniería Mecánica, Fundación Universidad de América, Bogotá D. C., Colombia.

Masina, N., Choonara, Y., Kumar, P., du Toit, L., Govender, M., Indermun, S., y Pillay, V. (2016). A review of the chemical modification techniques of starch. Carbohydrate Polymers, 157, $1226-1236$

Morris, V., Gunning, A., Faulds, C., Williamson, G., y Svensson, B. (2005). AFM images of complexes between amylose and Aspergillus niger glucoamylase mutants, native and mutant starch binding domains: A model for the action of glucoamylase. Starch/Staerke, 57, 1-7. https://doi.org/10.1002/star.200400333

Nowak, V., Du, J., y Charrondière, U. (2016). Assessment of the nutritional composition of quinoa (Chenopodium quinoa Willd.). Food Chemistry, 193, 47-54. https://doi.org/http://dx.doi. org/10.1016/j.foodchem.2015.02.111

Pastás, N., Bernal, C., y Pineda, Y. (2012). Desarrollo de un polvo cosmético compacto empleando octenilsuccinato alumínico de almidón de quinua (tesis de pregrado). Facultad de Ingeniero Química, Fundación Universidad de América, Bogotá D. C., Colombia.

Prego, I., Maldonado, S.,y Otegui,M. (1998). Seed Structure and Localization of Reserves in Chenopodium quinoa. Annals of Botany, 82, 481-488. https://doi.org/10.1006/anbo.1998.0704

Raghunathan, R., Hoover, R., Waduge, R., Liu, Q. y Warkentin, T.D. (2016). Impact of molecular structure on the physicochemical properties of starches isolated from different field pea (Pisum sativum L.) cultivars grow in Saskatchewan, Canada. Food Chemistry, 221, 15141521. https://doi.org/10.1016/j.foodchem.2016.10.142

Shevkani, K., Singh, N., Bajaj, R., y Kaur, A. (2017). Wheat starch production, structure, functionality and applications - a review. International Journal of Food Science and Technology, 52 (1), $38-58$ 
Souilah, R., Boudries, N., Djabali, D., Belhadi, B., y Nadjemi, B. (2015). Kinetic study of enzymatic hydrolysis of starch isolated from sorghum grain cultivars by various methods. Journal of Food Science and Technology, 52(1), 451-457. https://doi.org/10.1007/s13197-013-0977-z

Steffolani, M., León, A., y Pérez, G. (2013). Study of the physicochemical and functional characterization of quinoa and kaniwa starches. Starch/Staerke, 65(11-12), 1-8. https://doi. org/10.1002/star.201200286

Uthumporn, U., Shariffa, Y., y Karim, A. (2012). Hydrolysis of native and heat-treated starches at sub-gelatinization temperature using granular starch hydrolyzing enzyme. Applied Biochemistry and Biotechnology, 166, 1167-1182. https://doi.org/10.1007/s12010-011-9502-x

Varriano E. y Defrancisco, A. (1984). Ultrastructure of Quinoa Fruit (Chenopodium quinoa Willd). Food Microstructure, 3(2), 165-173. 